\title{
TWO CLASSES OF FRÉCHET-URYSOHN SPACES
}

\author{
ALAN DOW \\ (Communicated by Dennis Burke)
}

\begin{abstract}
Arhangel'skii introduced five classes of spaces, $\alpha_{i}$-spaces $(i<5)$, which are important in the study of products of Fréchet-Urysohn spaces. For each $i<5$, each $\alpha_{i}$-space is an $\alpha_{i+1}$-space and it follows from the continuum hypothesis that there are countable $\alpha_{i+1}$-spaces which are not $\alpha_{i}$-spaces. A $v$-space ( $w$-space) is a Fréchet-Urysohn $\alpha_{1}$-space $\left(\alpha_{2}\right.$-space). We show that there is a model of set theory in which each $\alpha_{2}$-space ( $w$-space) is an $\alpha_{1}$-space $(v$-space).
\end{abstract}

\section{INTRODUCTION}

Arhangel'skii defines a point $x \in X$ to be an $\alpha_{1}$-point ( $\alpha_{2}$-point) if whenever $F_{n}$ is a sequence converging to $x$, for each $n<\omega$, there is a sequence $F$ converging to $x$ such that $F_{n}-F$ is finite ( $F_{n} \cap F$ is infinite) for each $n<\omega$. Furthermore a point is called an $\alpha_{0}$-point if it has a countable neighbourhood base. A space is called an $\alpha_{1}$-space if each point is an $\alpha_{i}$-point. A space is Fréchet-Urysohn if whenever a point is in the closure of a set there is a sequence from it converging to the point.

Nyikos has shown that there is a countable $w$-space which is not first countable [Ny1, Ny2]. In [Ny2] Nyikos asks if there is a countable $w$-space which is not a $v$-space and a countable $v$-space which is not first-countable. Nyikos [Ny2] produces examples of countable $w$-spaces which are not $v$-spaces from a special set-theoretic assumption following from, for example, Martin's Axiom (even $\underline{b}<\underline{d}$ ). Nogura [N] has shown that MA (even $\underline{b}=\underline{d}$ ) implies there is an example of a countable $v$-space which is not first countable. It is shown in [DS] that it is consistent that each countable $v$-space is first-countable.

Gruenhage [G] introduced $w$-spaces and Sharma [Sh] obtained their characterization in terms of $\alpha_{2}$-spaces. The term $v$-space seems to be due to Nyikos.

Received by the editors July 18, 1988 and, in revised form, December 2, 1988.

1980 Mathematics Subject Classification (1985 Revision). Primary 54E35.

$K e y$ words and phrases. Fréchet-Urysohn space, $v$-space, $w$-space.

This research was supported by NSERC of Canada. 


\section{$\omega$-SPLITTING FAMILIES FROM $\alpha_{2}$-POINTS}

1. Definition. We say that $\mathfrak{X} \subset[\omega]^{\omega}$ is $\omega$-splitting if for each countable family $\left\{A_{n}: n \in \omega\right\} \subset[\omega]^{\omega}$ there is an $X \in \mathfrak{X}$ such that $\left|A_{n} \cap X\right|=\left|A_{n}-X\right|$ for each $n \in \omega$. We shall say that $X$ is $M$-splitting if $|A \cap X|=|A-X|$ for each $A \in M \cap[\omega]^{\omega}$.

Let $x$ be a point in a space $Y$ and suppose $F_{n} \in[Y]^{\omega}$ is a sequence converging to $x$ for each $n \in \omega$. Identify $\bigcup F_{n}$ with $\omega$ and define $\mathfrak{X}$ to be the set of $X$ which "think" that $x$ is an $\alpha_{1}$-point. That is, define $\mathfrak{X}\left(x,\left\langle F_{n}\right\rangle\right)=\left\{X \in[\omega]^{\omega}: \exists F \subset Y \quad\left(F\right.\right.$ converges to $x$ and $\left(X \cap F_{n}\right)-F$ is finite for each $n \in \omega)\}$. The definition of $\mathfrak{X}\left(x,\left\langle F_{n}\right\rangle\right)$ of course depends on the identification of $\bigcup F_{n}$ with $\omega$, but only up to a permutation on $\omega$ and this will never matter to us.

2. Lemma. If $F_{n} \in[Y]^{\omega}(n \in \omega)$ converges to an $\alpha_{2}$-point $x$, then $\mathfrak{X}=$ $\mathfrak{X}\left(x,\left\langle F_{n}\right\rangle\right)$ is an $\omega$-splitting family.

Proof. Assume that $\cup F_{n}$ is identified with $\omega$ and let $\left\{A_{k}: k \in \omega\right\} \subset[\omega]^{\omega}$. Let $I=\left\{j \in \omega: A_{j} \cap F_{n}\right.$ is finite for each $\left.n\right\}$. Choose for each $n$, a finite subset $H_{n}$ of $F_{n}-\bigcup_{m<n} F_{m}$ so that $A_{j}-H$ is finite for each $j \in I$ where $H=\bigcup H_{n}$. Now for each $j \in \omega-I$, choose $n_{j} \in \omega$ so that $A_{j} \cap F_{n_{j}}$ is infinite. Next choose an infinite $B_{j} \subset A_{j} \cap F_{n_{j}}$ so that $B_{j} \cap B_{k}=\varnothing$ for $j \neq k$ in $\omega-I$. Now since $x$ is an $\alpha_{2}$-point, there is a sequence $F$ converging to $x$ such that $F \cap B_{j}$ is infinite for each $j \in \omega-I$. It follows that $F \cup H$ hits each $A_{j}$ in an infinite set and that $F \cup H \in \mathfrak{X}$. Finally any infinite subset of $F \cup H$ is a member of $\mathfrak{X}$ hence there is an $X \in \mathfrak{X}$ which splits $\left\{A_{j}: j \in \omega\right\}$.

3. Lemma. If every $\omega$-splitting family contains an $\omega$-splitting family of cardinality less than $\underline{b}$, then each $\alpha_{2}$-point is an $\alpha_{1}$-point.

Proof. Let $x$ be an $\alpha_{2}$-point of a space $Y$ and assume $F_{n}$ is a sequence converging to $x$ for each $n \in \omega$. Since we wish to show that $x$ is an $\alpha_{1}$-point we may assume that the $F_{n}$ 's are pairwise disjoint and that $U F_{n}=\omega$. Let $\mathfrak{X}=\mathfrak{X}\left(x,\left\langle F_{n}\right\rangle\right)$ be defined as above. By Lemma 2 and the hypothesis of this lemma, there is an $\omega$-splitting family $\mathfrak{X}^{\prime} \in[\mathfrak{X}]^{<\underline{b}}$. For each $A \in \mathfrak{X}^{\prime}$, choose (by the definition of $\mathfrak{X}$ ) a sequence $F_{A}$ converging to $x$ and $f_{A} \in{ }^{\omega} \omega$ so that $\left(A \cap F_{n}\right)-F_{A} \subset f_{A}(n)$ for each $n \in \omega$. Now choose $f \in{ }^{\omega} \omega$ so that $f_{A}<^{*} f$ for each $A \in \mathfrak{X}^{\prime}$ which we may do since $\left|\mathfrak{X}^{\prime}\right|<\underline{b}$. We claim that $F=\bigcup F_{n}-f(n)$ converges to $x$, which would show that $x$ is an $\alpha_{1}$-point. Indeed, assume $F$ does not converge to $x$ and choose $F^{\prime} \in[F]^{\omega}$ such that $x$ is not a limit point of $F^{\prime}$. Since $\mathfrak{X}^{\prime}$ is splitting, choose $A \in \mathfrak{X}^{\prime}$ so that $A \cap F^{\prime}$ is infinite. However this contradicts that $F_{A}$ converges to $x$ since $A \cap F^{\prime}-F_{A}$ is finite. 
4. Remark. We could replace the notion of $\omega$-splitting in Lemma 3 by what one might call " $\omega$-hitting".

\section{$\omega$-SPLITTING FAMILIES AND LAVER FORCING}

5. Theorem. In a model obtained by adding $\omega_{2}$-Laver reals to a model of $\mathrm{CH}$, every $\omega$-splitting family contains an $\omega$-splitting family of cardinality $\omega_{1}$. Hence in this model each $\alpha_{2}$-space ( $w$-space) is an $\alpha_{1}$-space ( $v$-space).

The theorem follows from the following four results. Proposition 6 is a collection of standard facts about Laver forcing, Lemma 7 is a standard reflection argument, Lemma 8 is a special case of a general preservation scheme proven in [S2] and Lemma 9 is new. Recall that $T \in \mathrm{L}$ (the Laver poset defined in [L]) if $T \subset{ }^{<\omega} \omega$ has a root $t_{0}=\operatorname{root}(T)$ and for $t_{0} \leq t \in T \quad\left\{n: t^{\wedge} n \in T\right\}$ is infinite. $L$ is ordered by inclusion. For $\lambda \leq \omega_{2}$ let $\mathbb{P}_{\lambda}$ be the countable support $\lambda$-stage iteration of the forcing notion $L$.

6. Proposition. [CH] $\mathbb{P}_{\omega_{2}}$ is an $\omega_{2}-c c$ proper poset such that $1 \|-\mathbf{P}_{\lambda} \underline{b}=\underline{c}=$ $\left|\lambda \cdot \omega_{1}\right|^{V}$. Furthermore if $\mu<\lambda$ then $\mathbb{P}_{\lambda}$ is forcing isomorphic to $\mathbb{P}_{\mu} * \mathbb{P}_{\lambda}$.

For proofs of the various assertions in Proposition 6 we refer the reader to [L] and [S1].

7. Lemma. [CH] Let $\left\{X_{\alpha}: \alpha<\omega_{2}\right\}$ be $\mathbb{P}_{\omega_{2}}$-names such that $1 \|-\left\{X_{\alpha}: \alpha<\right.$ $\left.\omega_{2}\right\} \subset[\omega]^{\omega}$ is $\omega$-splitting, then there is a $\lambda<\omega_{2}$ such that $1 \|-\mathbf{P}_{\lambda}\left\{X_{\alpha}: \alpha<\lambda\right\}$ is $\omega$-splitting.

Notation. If $p$ is a member of a poset $P$ and $M$ is a set, " $p \|-_{P} X$ is $M$-splitting" will abbreviate $p \|-|A \cap X|=|A-X|$ for each $P$-name $A \in M$ such that $p \|-A \in[\omega]^{\omega}$.

We shall say that a poset $P$ is $\omega$-splitting if the following are satisfied: whenever $P \in M$, where $M$ is a countable elementary submodel of $H(\theta)$ for any sufficiently large $\theta, p \in M \cap P$ and $X$ is $M$-splitting then there is some $q<p$ which is $(M, P)$-generic and such that $q \|-X$ is $M$-splitting.

Note that an iteration of finitely many $\omega$-splitting posets is again $\omega$-splitting; hence proper.

8. Lemma. If $P_{\delta}=\left\langle\left(P_{\alpha}, Q_{\alpha}\right): \alpha<\delta\right\rangle \in M$ is a countable support iteration of $\omega$-splitting (hence proper) posets then $P_{\delta}$ is also $\omega$-splitting.

9. Lemma. Let $M$ be a countable elementary submodel of $H\left(\omega_{3}\right)$ and let $T \in$ $\mathrm{L} \cap M$, then if $X$ is $M$-splitting there is an $(M, \mathrm{~L})$-generic $T^{\prime}<T$ such that $T^{\prime} \|-X$ is $M$-splitting. Therefore $\mathrm{L}$ is $\omega$-splitting.

It may be worthwhile to record the following corollary to the above results.

10. Corollary. If a family is $\omega$-splitting then it will still be $\omega$-splitting after forcing with the countable support iteration of Laver forcing. Furthermore in any 
model obtained by adding iteratively $\omega_{2}$-Laver reals the splitting number, $\underline{s}$, will be $\omega_{1}$.

Before proving Lemmas 7-9 let us indicate how Theorem 5 now follows. Let $G$ be $\mathbf{P}_{\omega_{2}}$-generic over $V$ (a model of $\mathrm{CH}$ ). Let $\mathfrak{X}=\left\{X_{\alpha}: \alpha<\omega_{2}=\underline{c}\right\}$ be an $\omega$-splitting family. Choose $\mathbf{P}_{\omega_{2}}$-names $\left\{X_{\alpha}: \alpha<\omega_{2}\right\} \in V$ so that $1 \|-\mathfrak{X}=$ $\left\{X_{\alpha}: \alpha<\omega_{2}\right\}$. By Lemma 7, there is a $\lambda<\omega_{2}$ so that $1 \|{ }_{P_{\lambda}}\left\{X_{\alpha}: \alpha<\lambda\right\}$ is $\omega$-splitting. Let $G_{\lambda}=G \cap \mathbf{P}_{\lambda}$; hence $V\left[G_{\lambda}\right] \models\left\{X_{\alpha}: \alpha<\lambda\right\}$ is $\omega$-splitting. Since $\mathbf{P}_{\omega_{2}} \cong \mathbf{P}_{\lambda} * \mathbf{P}_{\omega_{2}}, V[G] \models\left\{X_{\alpha}: \alpha<\lambda\right\}$ is $\omega$-splitting by Lemmas 8 and 9 .

It remains to prove the Lemmas.

Proof of Lemma 7. By Proposition 6, we may assume that for each $\alpha<\omega_{2}$, there is an $f(\alpha)<\omega_{2}$ such that $X_{\alpha}$ is a $\mathbb{P}_{f(\alpha)}$-name. Also if $G$ is $\mathbb{P}_{\omega_{2}}$ generic, $V[G] \models$ for each $\alpha<\omega_{2}$, there is a $g(\alpha)<\omega_{2}$ such that for each set $M \in\left[[\omega]^{\omega}\right]^{\omega} \cap V\left[G_{\alpha}\right]$ there is an $X \in\left\{\operatorname{val}\left(X_{\beta}, G\right): \beta<g(\alpha)\right\}$ which is $M$-splitting (since $\left.V\left[G_{\alpha}\right] \models \underline{c}=\omega_{1}\right)$. Since $\mathbb{P}_{\omega_{2}}$ is $\omega_{2}-c c$ we may assume that $g \in V$. Now let $h$ be a continuous strictly increasing function from $\omega_{2}$ into $\omega_{2}$ such that $f(\alpha)+g(\alpha)<h(\alpha+1)$ for all $\alpha<\omega_{2}$. Choose $\lambda<\omega_{2}$ such that $h(\lambda)=\lambda$; it follows that $1 \|-\mathbf{P}_{\lambda}\left\{X_{\alpha}: \alpha<\lambda\right\}$ is $\omega$-splitting.

Proof of Lemma 8. Technically we make the inductive assumption that for each $\beta<\alpha<\delta$ and each $p \in P_{\beta}$ we have that $p \|-P_{\alpha} / P_{\beta}$ is $\omega$-splitting; where, as usual, $P_{\alpha} / P_{\beta}$ denotes the $P_{\beta}$-name satisfying the equation $P_{\alpha}=P_{\beta} *\left(P_{\alpha} / P_{\beta}\right)$. However in proving the inductive step we can just force with $P_{\beta}$ and work in the extension. Therefore we may make the above inductive assumption and complete the proof by showing that $P_{\delta}$ is $\omega$-splitting. As remarked when we defined the notion of an $\omega$-splitting poset the proof is trivial in case $\delta$ is a successor ordinal. Now let $\theta$ be a large enough cardinal (i.e. $\left.\left|\mathscr{P}\left(P_{\delta}\right)\right|<\theta\right)$ and let $P_{\delta} \in M$ where $M$ is a countable elementary submodel of $H(\theta)$. Suppose further that $p \in M \cap P_{\delta}$ and that $X$ is $M$-splitting. Let $\left\{A_{n}: n \in \omega\right\}$ index the set of $P_{\delta}$-names of subsets of $\omega$ which are in $M$. We may as well assume that $\operatorname{cf}(\delta)=\omega$ since we will be choosing $q$ to be $M$-generic and this $q$ will force that the $A_{n}$ 's are essentially $P_{M \cap \delta}$-names. We may therefore choose a countable increasing sequence of ordinals cofinal in $\delta$ and by our inductive assumption we may as well assume that $\delta=\omega$.

As in [S2], we shall choose sequences $p_{n}, q_{n}, k_{n}$ and $m_{n}$, by induction on $n$, so that:

(1) $p_{n} \in P_{\omega}, q_{n} \in P_{n}$ and $k_{n}, m_{n}$ are $P_{\omega}$-names of integers,

(2) $p_{n+1}\left|n=p_{n}\right| n, p_{n+1}<p_{n}, q_{n+1} \mid n=q_{n}$ and $q_{n}<p_{n} \mid n$,

(3) for $n>0, q_{n} \wedge p_{n} \|-A_{n}$ is finite or $m_{n} \in X \cap A_{n}$ and $k_{n} \in A_{n}-X$, $\left(q_{n} \wedge p_{n}\right.$ is meant to denote the element $\left.\left(q_{n} \wedge p_{n} \mid n\right)^{\wedge} p \mid[n, \omega) \in P_{\omega}\right)$

(4) $q_{n}$ is $\left(M, P_{n}\right)$-generic and $q_{n} \|-_{P_{n}} X$ is $M$-splitting, and

(5) $q_{n} \|-p_{n} \in M$.

Before we begin the induction, let us comment on what (5) means. It is not the case that $p_{n}$ will be in $M$ but $q_{n} \|-\left(\exists p^{\prime} \in M\right)$ such that $p^{\prime} / P_{n}=p_{n} / P_{n}$; 
where $p / P_{n}$ denotes the $P_{\omega} / P_{n}$-name corresponding to $p$ in the extension by $P_{n}$. It is not even the case that we can bring the " $\exists$ " sign outside the forcing statement. However condition 5 is essential in order for the induction to continue. Let $p_{1}=p$ where $p \in P_{\omega} \cap M$ is as chosen above. Since $P_{1}$ is proper (and we have chosen $\theta$ large enough) we may choose $q_{1}<p_{1} \mid 1$ to be $\left(M, P_{1}\right)$-generic such that $q_{1} \|-X$ is $M$-splitting. Suppose that $p_{n}$ and $q_{n}$ have been chosen. Let $G_{n}$ be $P_{n}$-generic such that $q_{n}$ and $p_{n} \mid n$ are in $G_{n}$. In $V\left[G_{n}\right]$, let $p_{n}^{\prime}$ be the element of $M$ so that $p_{n}^{\prime} / P_{n}=p_{n} / P_{n}$, and let $B_{n+1}=\left\{m:\left(\exists p \in P_{\omega} / P_{n}\right) \quad p<p_{n}^{\prime} / P_{n}\right.$ and $\left.p \|-m \in A_{n+1}\right\}$. Here we are assuming that $A_{n} / P_{n}$ is the $P_{\omega} / P_{n}$-name which results from evaluating the $P_{\omega}$-name $A_{n}$ and similarly for $p_{n}^{\prime}$. Now $M\left[G_{n}\right]$ is an elementary submodel of $H(\theta)^{V\left[G_{n}\right]}$ which is a model of ZF-P (see [S1]) and $p_{n}^{\prime} \in M$, hence $B_{n+1} \in M\left[G_{n}\right]$. Since $X$ is $M\left[G_{n}\right]$-splitting we may choose $m_{n+1} \in X \cap B_{n+1}$ and $p^{\prime} / P_{n}<p_{n}^{\prime} / P_{n}$, $p^{\prime} \in M$ (by elementarity) such that $p^{\prime} \|-m_{n+1} \in A_{n+1}$. Similarly we may choose $p_{n+1}^{\prime} / P_{n}<p^{\prime} / P_{n}$ and $k_{n+1} \notin X$ such that $p_{n+1}^{\prime} \in M, p_{n+1}^{\prime} \mid n \in G_{n}$ and $p_{n+1}^{\prime} \|-k_{n+1} \in A_{n+1}$. By assumption $Q_{n}$ is $\omega$-splitting (in $V\left[G_{n}\right]$ ) hence we may choose $q_{n+1}^{\prime}<p_{n+1}^{\prime}(n), q_{n+1}^{\prime} \in Q_{n}$ so that $q_{n+1}^{\prime}$ is $\left(M\left[G_{n}\right], Q_{n}\right)$-generic and so that $q_{n+1}^{\prime} \|-X$ is $M\left[G_{n}\right]$-splitting. Now we use the maximality principle to choose $q_{n+1}, p_{n+1}$ and the names $m_{n+1}$ and $k_{n+1}$ so as to satisfy (1)-(5). That is, $p_{n} \mid n \|-$ if $X$ is $M\left[G_{n}\right]$-splitting and $M\left[G_{n}\right]$ is an elementary submodel of $H(\theta)^{V\left[G_{n}\right]}$ then there are $p_{n+1}^{\prime} / P_{n} \in M\left[G_{n}\right], k_{n+1}$ and $m_{n+1}$ as above. So we may choose a $P_{n}$-name, say $p_{n+1}^{\prime \prime}$, of an element of $P_{\omega} / P_{n}$ and $P_{\omega}$-names $k_{n+1}$ and $m_{n+1}$ so that $p \mid n \|-$ if $X$ is $M\left[G_{n}\right]$-splitting and $M\left[G_{n}\right]$ is an elementary submodel of $H(\theta)^{V\left[G_{n}\right]}$ then $p_{n+1}^{\prime \prime} \in M\left[G_{n}\right]$ and $p_{n+1}^{\prime \prime} \|-k_{n+1}$ and $m_{n+1}$ are as above. Next we choose a $P_{n}$-name $q_{n+1}$ for $q_{n+1}^{\prime}$. We let $p_{n+1}=p_{n} \wedge p_{n+1}^{\prime \prime}$ and $q_{n+1}=q_{n} \wedge q_{n+1}$. It is clear that (1), (2) are satisfied. By [S1], $q_{n+1}$ is $\left(M, P_{n+1}\right)$-generic and clearly $q_{n+1} \|-X$ is $M$-splitting; hence (4) holds. The reason that (3), (5) are satisfied is that $q_{n+1} \|-M\left[G_{n+1}\right]$ is an elementary submodel of $H(\theta)^{V\left[G_{n+1}\right]}$, hence $p_{n+1}^{\prime \prime}$ has the desired properties.

Now if $q \in P_{\omega}$ is such that $q \mid n=q_{n}$ for each $n \in \omega$ then $q$ is $\left(M, P_{\omega}\right)$ generic (see [S1]) and $q<p_{n}$ for each $n \in \omega$. It follows that $q \|-X$ is $M$ splitting, since for each $i<j<\omega$ there is an $n<\omega$ such that $1 \|-A_{n}=A_{i}-j$ and so $q<q \mid n \wedge p_{n} \|-m_{n} \in X \cap A_{i}-j$ and $k_{n} \in A_{i}-(X \cup j)$.

Proof of Lemma 9. Let $T \in L \cap M$ where $M$ is a countable elementary submodel of $H\left(\omega_{s}\right)$ and assume $X$ is $M$-splitting. Fix indexings $\left\{A_{n} \mid n \in \omega\right\}$ of $M \cap\left\{A \mid A\right.$ is an $L$-name and $\left.1 \vDash A \in[\omega]^{\omega}\right\}$, and $\left\{D_{n} \mid n \in \omega\right\}$ of $M \cap\{D \subseteq \mathbf{L} \mid D$ is dense open $\}$. We shall inductively define a descending sequence $\left\{T_{n} \mid n \in \omega\right\} \subset \mathbf{L}$ (with $T_{0}=T$ ) so that, for each $n \in \omega$ :

1. $T_{n} \cap^{n} \omega=T_{n+1} \cap^{n} \omega$, and

2. If $T^{\prime}<T_{n}$ then there is a $t \in T^{\prime}$ such that

(a) $\left(T_{n}\right)_{t} \in M \cap D_{n}$ and 
(b) $\left(T_{n}\right)_{t} \vDash X \cap A_{n} \neq \varnothing$ and $A_{n}-X \neq \varnothing$.

If we accomplish this, then condition 1 guarantees that $T^{\prime}=\cap_{n} T_{n} \in L$. It is easy to see that condition 2(b) guarantees that $T^{\prime} \models X$ is $M$-splitting. Condition 2(a) actually has the double role of ensuring that $T^{\prime}$ is $M$-generic and allowing the induction to continue.

Following [L], if $S^{\prime}, S \in \mathbf{L}$ then we use $S^{\prime}<{ }^{0} S$ to denote the situation where $S^{\prime}<S$ and they have the same root. One of the key facts about Laver forcing from [L] is that if $S \in \mathbf{L}$ and $\varphi$ is any sentence of the forcing language then there is an $S^{\prime}<^{0} X$ such that either $S^{\prime} \models \varphi$ or $S^{\prime} \models \neg \varphi$. Therefore

$$
(*)=\left\{\begin{array}{l}
\text { if } A \text { is an } L \text {-name and } F \text { is a finite set such that } \\
S \models F \cap A \neq \varnothing, \text { then there is an } S^{\prime}<{ }^{0} S \text { and an } x \in F \\
\text { such that } S \models x \in A .
\end{array}\right.
$$

Let us assume that $0<n \in \omega$ and that $T_{n-1}$ has been chosen as above. Let $I$ be the set of members of $T_{n-1}-\leq n \omega$ which are minimal with respect to the property that $\left(T_{n-1}\right)_{t} \in M$. Note that the minimality of the members of $I$ and condition 2(a) guarantee that the collection $\left\{\left(T_{n-1}\right)_{t} \mid t \in I\right\}$ is an antichain in $\mathbf{L}$ which is maximal below $T_{n-1}$. Now if we find, for each $t \in I$, a condition $T_{t}^{\prime}<{ }^{0}\left(T_{n-1}\right)_{t}$ satisfying condition 2 , then we can define $T_{n}$ to be $\bigcup\left\{T_{t}^{\prime} \mid t \in I\right\}$. This works since $\left\{T_{t}^{\prime} \mid t \in I\right\}$ is a maximal-below- $T_{n}$ antichain. For the same reason, repeated uses of Facts 1 to 3 finish the proof.

Fact 1. If $S \in \mathbf{L} \cap M$ and $n \in \omega$, then there is an $S^{\prime}<^{0} X$ such that the collection $\left.\left(S^{\prime}\right)_{t} \mid\left(S^{\prime}\right)_{t} \in D_{n} \cap M\right\}$ is predense below $S^{\prime}$.

Fact 2. If $S \in \mathbf{L} \cap M$ and $n \in \omega$ there is an $S^{\prime}<{ }^{0} X$ such that the collection $\left\{\left(S^{\prime}\right)_{t}:\left(S^{\prime}\right)_{t} \in M\right.$ and $\left.\left(S^{\prime}\right)_{t} \vDash X \cap A_{n} \neq \varnothing\right\}$ is predense below $S^{\prime}$.

Fact 3. If $S \in \mathbf{L} \cap M$ and $n \in \omega$ there is an $S^{\prime}<{ }^{0} S$ such that the collection $\left\{\left(S^{\prime}\right)_{t} \mid\left(S^{\prime}\right)_{t}\right.$ and $\left.\left(S^{\prime}\right)_{t} \vDash A_{n}-X \neq \varnothing\right\}$ is predense below $S^{\prime}$.

Fact 1 is, of course, a well-known property of $\mathbf{L}$ and its proof is similar to the proof of Fact 2. In the proof of Fact 2 we are just using that $X$ is $M$-splitting. Since $\omega-X$ is also $M$-splitting, Fact 3 follows from Fact 2. Now let us prove Fact 2.

Let $B_{n}=\left\{k \in \omega \mid\left(\exists S^{\prime}<^{0} S\right) S^{\prime} \models k \in A_{n}\right\}$. Let us first suppose that $B_{n}$ is infinite. In this case, $B_{n} \in[\omega]^{\omega} \cap M$, hence we have that $X \cap B_{n} \neq \varnothing$. It follows that we may choose $S^{\prime}<^{0} S, S^{\prime} \in M$ and $k \in X \cap B_{N}$ so that $S^{\prime} \vDash k \in A_{n}$-which certainly suffices.

Now let us suppose that $\max \left(B_{n}\right)<m$. Let $I$ be the set of minimal elements of $\left\{t \in S \mid\left(\exists m_{t} \geq m\right)\left(\exists S_{t}^{\prime}<^{0} X_{t}\right) S_{t}^{\prime} \models m_{t} \in A_{n}\right\}$. Since $\left.1 \models(\exists k \geq m)\right) k \in A_{n}$ and the members of $I$ are minimal, $\left\{S_{t} \mid t \in I\right\}$ is a maximal-below- $S$ antichain of $\mathbf{L}$. For each $t \in I$, fix a minimal $m_{t}$ and an $S_{t}^{\prime} \in M$ as in the description of $I$; hence $\left\{m_{t} \mid t \in I\right\} \in M$. We shall show that $S^{\prime}=\bigcup\left\{S_{t}^{\prime} \mid t \in I\right.$ and $\left.m_{t} \in X\right\}$ works. That is, we prove that $S^{\prime} \in \mathbf{L}, S^{\prime}<^{0} S$ and simply note that $\left\{S_{t}^{\prime} \mid t \in I\right.$ and $\left.m_{t} \in X\right\}$ is a maximal-below- $S^{\prime}$ antichain. Therefore it suffices to show 
that if $s \in S^{\prime}$ is such that $\operatorname{root}(S) \geq s$, then $s$ has infinitely many immediate successors in $S^{\prime}$. First suppose that there is a $t \in I$ with $t \leq s$. Since members of $I$ are minimal, it follows that $\left(S^{\prime}\right)_{s}=S^{\prime} \cap S_{s}$-hence $t \in S^{\prime}$ and $m_{t} \in X$. Therefore $S_{t}^{\prime} \subseteq S^{\prime}$ and $s$ has infinitely many immediate successors in $S^{\prime}$. If there is no such $t \in I$, then $S_{s}$ has no $<^{0}$-extension which decides a value of $A_{n}$ above $m$. Suppose now that $k \in \omega$ is such that $\max \left(\left\{i \mid s^{\wedge} i \in S^{\prime}\right\}\right)<k$. Let $S^{\prime \prime}$ be the $<^{0}$-extension of $S_{s}$ obtained by removing $\left\{t \mid(\exists i<k) s^{\wedge} i \leq t\right\}$. We claim that $\left\{m_{t} \mid t \in I \cap S^{\prime \prime}\right\}$ is infinite. Indeed, since $\left\{S_{t} \mid t \in I \cap S^{\prime \prime}\right\}$ is predense below $S^{\prime \prime}, S^{\prime \prime} \models A_{n} \cap\left\{m_{t} \mid t \in I \cap S^{\prime \prime}\right\} \neq \varnothing$. If the set was finite then, by $(*), S^{\prime \prime}$ (hence $S_{s}$ ) would have $<^{0}$-extension picking one of the values. But now $X \cap\left\{m_{t} \mid t \in I \cap S^{\prime \prime}\right\}$ is nonempty, hence any $t \in I \cap S^{\prime \prime}$ with $m_{t} \in X$ is an extension of $s$ in $S^{\prime}$-a contridiction to the choice of $k$.

\section{REFERENCES}

[A1] A. V. Arhangel'skii, The frequency spectrum of a topological space and the classification of spaces, Dokl. Akad. Nauk. SSSR 206 (1972), 265-268 = Sov. Math. Dokl. 13 (1972), 265268.

[A2] _ - The frequency spectrum of a topological space and the product operation, Trudy Mosk. Mat. Obs. 40 (1979) = Transl. Moscow Math. Soc. (1981), Issue 2, 163-200.

[DS] A. Dow and J. Steprāns.

[G] G. Gruenhage, Infinite games and generalizations of first countable spaces, Gen. Top. Appl. 6 (1976), 339-352.

[L] R. Laver, On the consistency of Borel's conjecture, Acta Math. 137 (1976), 151-169.

[LNy] R. Levy and P. Nyikos, Families in $\beta \omega$ whose union is regular open, preprint.

[N] T. Nogura, A counterexample for a problem of Arhangel'skii concerning the product of Fréchet spaces, Top. Appl. 25 (1987), 75-80.

[Ny1] P. Nyikos, ${ }^{\omega} \omega$ and the Fréchet-Urysohn property, in preparation.

[Ny2] _ The Cantor tree and the Fréchet-Urysohn property, preprint, 1987.

[O] R. C. Olson, Bi-quotient maps and countably bisequential spaces, Gen. Top. Appl. 4 (1974), $1-28$.

[S1] S. Shelah, Proper forcing, Springer Lecture Notes, 1982.

[S2] __ Cardinal invariants of the continuum, Axiomatic Set Theory, Eds. J. Baumgartner, D. A. Martin, S. Shelah, Contemp. Math. Amer. Math. Soc., Providence, R.I., 1986.

[Sh] Sharma, Some characterizations of $W$-spaces and w-spaces, Gen. Top. Appl. 9 (1978) 289293.

Department of Mathematics, York University, North York, Ontario, M3J 1P3, CANADA 